H. Yoshida

Nagoya Math. J.

Vol 46 (1972), 111-120

\title{
TANGENTIAL BOUNDARY PROPERTIES OF ARBITRARY FUNGTIONS IN THE UNIT DISG
}

\author{
HIDENOBU YOSHIDA
}

1. By the method of Dolzhenko's paper, we studied relations between non-tangetial (angular) boundary behaviors and horocyclic boundary behaviors of arbitrary functions defined in the open unit disc of the complex plane in [8]. Vessey [5], [6] investigated the behavior of arbitrary functions on paths which are "more tangential" than horocycles. The purpose of the present paper is to prove the fact that is sharper than the results in Vessey [5], [6], and generalize the results in [8] to obtain the connection between behaviors on two "more tangential" angles.

Notations and definitions. Unless otherwise stated, $f: D \rightarrow W$ shall denote an arbitrary function (generally not one-valued) defined in the open unit disc $D:\{z ;|z|<1\}$ and assuming values in the extended complex plane $W$. The unit circle $\{z ;|z|=1\}$ is denoted by $\Gamma$.

Suppose a set $A \subset \Gamma$ and a point $\zeta=e^{i \theta} \in \Gamma$ are given. For an $\varepsilon>0$, we denote an arc $\left\{e^{i \theta^{\prime}} ; \theta-\varepsilon<\theta^{\prime}<\theta+\varepsilon\right\}$ by $\Gamma(\varepsilon, \zeta)$. Let $\gamma(\zeta, \varepsilon, A)$ be the largest length of arcs contained in $\Gamma(\varepsilon, \zeta)$ and not intersecting with $A$. The set $A$ is of porosity of the order $\mu, 0<\mu \leqq 1$ (or simply of porosity $(\mu)$ ) at $\zeta$, if

$$
\varlimsup_{\varepsilon \rightarrow 0} \frac{1}{\varepsilon}\{\gamma(\zeta, \varepsilon, A)\}^{\mu}>0 .
$$

A is of porosity $(\mu)$ on $\Gamma$ if it is so at each $\zeta \in A$. A set which is a countable sum of sets of porosity $(\mu)$ is said to be of $\sigma$-porosity $(\mu)$.

A set of $\sigma$-porosity $(\mu)$ is of the first Baire category.

It is easily seen that a measurable set which is of porosity (1) on $\Gamma$ has no points of density. Therefore every measurable set of $\sigma$-porosity (1) on $\Gamma$ is of measure 0 . But there exists the set, which is of measure 0 and not of $\sigma$-porosity (1) (see [7], p. 75).

Received May 28, 1971. 
For $0 \leqq q<\infty, 0<\alpha<\infty$ and $\zeta=e^{i \theta} \in \Gamma$, we denote by $t^{+}(\alpha, q)(\zeta)$ the curve $\left\{z ; \alpha|\arg (z)-\theta|^{q+1}=1-|z|, \arg (z)>\theta\right\}$ terminating at $\zeta$ and by $t^{-}(\alpha, q)(\zeta)$ the reflection of $t^{+}(\alpha, q)(\zeta)$ with respect to the radius at $\zeta$.

For $0 \leqq q<\infty, 0<\alpha<\beta<\infty, 0<\delta<1$ and $\zeta \in \Gamma$, we define a right $q$-angle $\nabla^{+}(\alpha, \beta, \delta, q)(\zeta)$ as the open region lying between the curves $t^{+}(\alpha, q)$

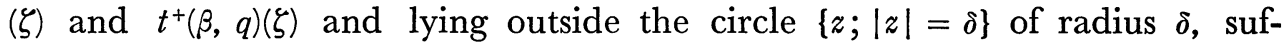
ficiently near to 1 . The left $q$-angle at $\zeta$ with parameters $(\alpha, \beta, \delta)$, denoted by $\nabla^{-}(\alpha, \beta, \delta, q)(\zeta)$, is the reflection of $\nabla^{+}(\alpha, \beta, \delta, q)(\zeta)$ with respect to the radius at $\zeta$. When convenient, we use the shorter notation $\nabla^{+}(q)(\zeta), \nabla^{-}(q)(\zeta)$ or $\nabla(q)(\zeta)$ without specifying whether it be right or left.

For $0 \leqq q<\infty, 0<\alpha<\infty, 0<\delta<1$ and $\zeta \in \Gamma$, we define a $q$-cycle $\Omega(\alpha$, $\delta, q)(\zeta)$ as the open region lying between the curves $t^{+}(\alpha, q)(\zeta)$ and $t^{-}(\alpha, q)(\xi)$ and lying outside the circle $\{z ;|z|=\delta\}$ of radius $\delta$, sufficiently near to 1 . When convenient, we use the shorter notation $\Omega(q)(\zeta)$ without specifying $\alpha$.

If $f: D \rightarrow W$ and $\zeta \in \Gamma$, we can define, in the usual manner, the cluster sets at $\xi$ on the sets $\nabla^{+}(\alpha, \beta, \delta, q)(\zeta), \nabla^{-}(\alpha, \beta, \delta, q)(\zeta)$ and $\Omega(\alpha, \delta, q)(\zeta)$.

We denote by $E_{\nabla(q) \nabla(q)}(f)$ the set of points $\zeta \in \Gamma$ such that $C_{\nabla(q)(\zeta)}(f, \zeta) \neq$ $C_{\nabla /(q)(\xi)}(f, \zeta)$ for some pair of two $q$-angles $\nabla(q)(\zeta)$ and $\nabla^{\prime}(q)(\zeta)$. The complement of $E_{\nabla(q) \nabla(q)}(f)$ with respect to $\Gamma$ is denoted by $K_{q}(f)$.

We denote by $E_{\Omega\left(q_{1}\right) \Omega\left(q_{2}\right)}(f)$ the set of points $\zeta \in \Gamma$ such that $C_{\Omega\left(q_{1}\right)(\xi)}(f, \zeta)$ $\neq C_{\Omega\left(q_{2}\right)(\xi)}(f, \zeta)$ for some pair of $q_{1}$-cycle $\Omega\left(q_{1}\right)(\zeta)$ and $q_{2}$-cycle $\Omega\left(q_{2}\right)(\zeta)$.

We denote by $E_{\Omega(q) \nabla(q)}(f)$ the set of points $\zeta \in \Gamma$ such that $C_{\Omega(q)(\xi)}(f, \zeta) \neq$ $C_{\nabla(q)(\xi)}(f, \zeta)$ for some pair of $q$-cycle $\Omega(q)(\zeta)$ and $q$-angle $\nabla(q)(\zeta)$.

A point $\zeta \in \Gamma$ is said to be a $q$-angular Plessner point (or a $q$-cyclic Plessner point) of $f(z)$ provided that

$$
C_{\nabla^{+}(q)(\xi)}(f, \zeta)=W \text { and } C_{\nabla^{-}(q)(\xi)}(f, \zeta)=W\left(\text { or } C_{\Omega(q)(\xi)}(f, \zeta)=W\right)
$$

for each right and left $q$-angle (or $q$-cycle) at $\zeta$.

A point $\zeta \in \Gamma$ is called a $q$-angular Fatou point (or $q$-cyclic Fatou point) of $f(z)$ with a $q$-angular Fatou value (or $q$-cyclic Fatou value) $w \in W$ provided that

$$
\left.C_{\nabla^{+}(q)(\zeta)}(f, \zeta)=\{w\} \text { and } C_{\nabla^{-}(q)(\zeta)}(f, \zeta)=\{w\} \text { (or } C_{Q(q)(\zeta)}(f, \zeta)=\{w\}\right)
$$

for each right and left $q$-angle (or $q$-cycle) at $\zeta$.

2. Let $\left\{\alpha_{i}\right\},\left\{\beta_{i}\right\}$ and $\left\{\delta_{i}\right\}$ be sequences of all rational numbers satisfying $0<\alpha_{i}, \beta_{i}<\infty, 0<\delta_{i}<1$ and $\left\{D_{n}\right\}$ be a sequence consisting of all closed 
circles of the plane $W$ having rational radii and centers with rational coordinates. For an $\varepsilon>0$, we set $U_{\varepsilon}(\zeta)=\{z ;|z-\zeta|<\varepsilon\}$.

Lemma 1. Let $\zeta \in A \subset \Gamma$ and $0 \leqq q_{1} \leqq q_{2}$. Suppose $A$ is not of porosity $\left(\frac{q_{1}+1}{q_{2}+1}\right)$ at a point $\zeta \in A$. Then for fixed positive integers $s, t, k$ and $l, \Omega\left(\alpha_{s}\right.$, $\left.\delta_{t}, q_{2}\right)(\zeta) \cap U_{\varepsilon}(\zeta)$ is covered by the set $M=\underset{\xi \in A}{\cup} \Omega\left(\alpha_{k}, \delta_{l}, q_{1}\right)(\xi)$ supposed $\varepsilon$ is sufficiently small.

Proof. Without loss of generality, we may assume that $\zeta=1$. Now we suppose that there exists a sequence $z_{\nu}=r_{\nu} e^{i \theta_{\nu}}(\nu=1,2,3, \cdots)$ such that $z_{\nu} \in \Omega\left(\alpha_{s}, \delta_{t}, q_{2}\right)(1), z_{\nu} \notin M$ and $z_{\nu} \rightarrow 1$. For each $z_{\nu}$, points $R\left(z_{\nu}\right)$ and $S\left(z_{\nu}\right)$ on $\Gamma$ are decided as follows.

$R\left(z_{\nu}\right)$ is the point on $\Gamma$ such that the point $z_{\nu}$ lies on the curve $t^{+}\left(\alpha_{k}\right.$, $\left.q_{1}\right)\left(R\left(z_{\nu}\right)\right) . \quad S\left(z_{\nu}\right)$ is the point on $\Gamma$ such that the point $z_{\nu}$ lies on the curve $t^{-}\left(\alpha_{k}, q_{1}\right)\left(S\left(z_{\nu}\right)\right)$.

We immediately have

$\overline{R\left(z_{\nu}\right) S\left(z_{\nu}\right)}$ (the arc length connecting $R\left(z_{\nu}\right)$ and $\left.S\left(z_{\nu}\right)\right)=2\left(\frac{1-r_{\nu}}{\alpha_{k}}\right)^{\frac{1}{q_{1}+1}}$

$$
\begin{aligned}
& \overline{R\left(z_{\nu}\right) 1}=\left|\theta_{\nu}-\left(\frac{1-r_{\nu}}{\alpha_{k}}\right)^{\frac{1}{q_{1}+1}}\right|, \\
& \overline{S\left(z_{\nu}\right) 1}=\left|\theta_{\nu}+\left(\frac{1-r_{\nu}}{\alpha_{k}}\right)^{\frac{1}{q_{1}+1}}\right| .
\end{aligned}
$$

Since $z_{\nu} \in \Omega\left(\alpha_{s}, \delta_{t}, q_{2}\right)(1)$, we have

$$
\left|\theta_{\nu}\right|<\left(\frac{1-r_{\nu}}{\alpha_{s}}\right)^{\frac{1}{q_{2}+1}} .
$$

We set $\varepsilon_{\nu}=\max \left\{\overline{R\left(z_{\nu}\right) 1}, \overline{S\left(z_{\nu}\right) 1}\right\}$. Then we have

$$
\lim _{\nu \rightarrow \infty} \frac{\overline{R\left(z_{\nu}\right) S\left(z_{\nu}\right)}}{\left(1-r_{\nu}\right)^{\frac{1}{q_{1}+1}}}>0 \text { and } \varepsilon_{\nu}=O\left(\left(1-r_{\nu}\right)^{\frac{1}{q_{2}+1}}\right) \text { as } \nu \rightarrow \infty \text {. }
$$

Since $\left\{R\left(z_{\nu}\right) S\left(z_{\nu}\right)\right.$ (the arc connecting $R\left(z_{\nu}\right)$ and $\left.\left.S\left(z_{\nu}\right)\right)\right\} \cap A=\phi$, we have $r(1$, $\left.\varepsilon_{\nu}, A\right) \geqq \overline{R\left(z_{\nu}\right) S\left(z_{\nu}\right)}$. Thus we obtain

$$
\begin{aligned}
& \varlimsup_{\varepsilon \rightarrow 0} \frac{1}{\varepsilon}\{r(1, \varepsilon, A)\}^{\frac{q_{1}+1}{q_{2}+1}} \geqq \varlimsup_{\nu \rightarrow \infty} \frac{1}{\varepsilon_{\nu}} \overline{R\left(z_{\nu}\right) S\left(z_{\nu}\right)^{\frac{q_{1}+1}{q_{2}+1}}} \\
& \geqq \varlimsup_{\nu \rightarrow \infty} \frac{\left(1-r_{\nu}\right)^{\frac{1}{q_{2}+1}}}{\varepsilon_{\nu}}\left(\frac{\overline{R\left(z_{\nu}\right) S\left(z_{\nu}\right)}}{\frac{1}{\left(1-r_{\nu}\right)^{\frac{q_{1}+1}{q_{1}+1}}}}\right)^{\frac{q_{2}+1}{1}}>0,
\end{aligned}
$$


and this contradicts the assumption that 1 is not a point of porosity $\left(\frac{q_{1}+1}{q_{2}+1}\right)$ for $A$. Therefore, for $\varepsilon>0$ small enough, $\Omega\left(\alpha_{s}, \delta_{t}, q_{2}\right)(1) \cap U_{\varepsilon}(1)$ is covered by the set $M=\underset{\xi \in A}{\cup} \Omega\left(\alpha_{k}, \delta_{l}, q_{1}\right)(\xi)$.

THEOREM 1. Let $f: D \rightarrow W$ and $0 \leqq q_{1} \leqq q_{2}$. Then $E_{Q\left(q_{1}\right) \Omega\left(q_{2}\right)}(f)$ is of type $G_{\delta \sigma}$ and of $\sigma$-porosity $\left(\frac{q_{1}+1}{q_{2}+1}\right)$.

Proof. For positive integers $n, k$ and $l, E_{n, k, l}$ is the set of points $\zeta \in \Gamma$ such that

$$
\begin{aligned}
& \text { the set }\left\{w=f(z) ; z \in \Omega\left(\alpha_{k}, \delta_{l}, q_{1}\right)(\zeta)\right\} \\
& \text { lies at a distance } \geqq r_{n} \text { from } D_{n} .
\end{aligned}
$$

For positive integers $n, s, t$ and $u, F_{n, s, t, u}$ is the set of points $\zeta \in \Gamma$ such that

$$
\text { the set }\left\{w=f(z) ; z \in \Omega\left(\alpha_{s}, \delta_{t}, q_{2}\right)(\zeta), \frac{1}{3 u}<\operatorname{dis}(z, \Gamma)<\frac{1}{u}\right\}
$$

has common points with $D_{n}$.

Then $E_{n, k, l}$ is closed and $F_{n, s, t, u}$ is open. We put

$$
F_{n, s, t}=\bigcap_{i=1}^{\infty} \bigcup_{u=i}^{\infty} F_{n, s, t, u}
$$

and

$$
A_{n, k, l, s, t}=E_{n, k, l} \cap F_{n, s, t} .
$$

We will show

$$
E_{\Omega\left(q_{1}\right) \Omega\left(q_{2}\right)}(f)=\bigcup_{n, k, l, s, t} A_{n, k, l, s, t} .
$$

Take a point $\zeta \in E_{\Omega\left(q_{1}\right) \Omega\left(q_{2}\right)}(f)$. There exist $\Omega\left(q_{1}\right)(\zeta)$ and $\Omega\left(q_{2}\right)(\zeta), \Omega\left(q_{2}\right)(\zeta)$ $\supset \Omega\left(q_{1}\right)(\zeta)$, for which $C_{\Omega\left(q_{2}\right)(\xi)}(f, \zeta) \supsetneq C_{\Omega\left(q_{1}\right)(\xi)}(f, \zeta)$. Choose numbers $s, t$ and $j$ such that $\Omega\left(\alpha_{s}, \delta_{t}, q_{2}\right)(\zeta) \supset \Omega\left(q_{2}\right)(\zeta)$ and

$$
D_{j} \cap C_{Q\left(\alpha_{s}, \tilde{\delta}_{l}, q_{2}\right)(\zeta)}(f, \zeta) \neq \phi, \operatorname{dis}\left(D_{j}, C_{Q\left(q_{1}\right)(\zeta)}(f, \zeta)\right)>5 r_{j} .
$$

Then we can find positive integers $k$ and $l$ such that $\Omega\left(q_{1}\right)(\zeta) \supset \Omega\left(\alpha_{k}, \delta_{l}, q_{1}\right)(\zeta)$ and $\operatorname{dis}\left(D_{j}, f(z)\right)>4 r_{j}$ for $z \in \Omega\left(\alpha_{k}, \delta_{l}, q_{1}\right)(\zeta)$. If $D_{n}$ is a disc with radius $r_{n}$ $=2 r_{j}$ and concentric with $D_{j}$, $\operatorname{dis}\left(D_{n}, f(z)\right)>r_{n}$ for $z \in \Omega\left(\alpha_{k}, \delta_{l}, q_{1}\right)(\zeta)$, which shows $\zeta \in E_{n, k, l}$. In view of (6) there exists an infinite number of positive integers $u$ such that 


$$
D_{n} \cap\left\{w=f(z) ; z \in \Omega\left(\alpha_{s}, \delta_{t}, q_{2}\right)(\zeta), \frac{1}{3 u}<\operatorname{dis}(z, \Gamma)<\frac{1}{u}\right\} \neq \phi,
$$

which shows $\zeta \in F_{n, s, t}$. Thus $\zeta \in A_{n, k, l, s, t}$ and

$$
E_{\Omega\left(q_{1}\right) \Omega\left(q_{2}\right)}(f) \subset \bigcup_{n, k, l, s, t} A_{n, k, l, s, t}
$$

Take a point $\zeta \in \bigcup_{n, k, l, s, t} A_{n, k, l, s, t}$. If $\zeta \in A_{n, k, l, s, t}$,

$$
C_{Q\left(\alpha_{k}, \delta_{l}, q_{1}\right)(\xi)}(f, \zeta) \cap D_{n}=\phi \text { from }(1)
$$

and

$$
C_{\Omega\left(\alpha_{s}, \delta_{t}, q_{2}\right)(\xi)}(f, \zeta) \cap D_{n} \neq \phi \text { from }(2)
$$

Thus we have

$$
C_{Q\left(\alpha_{s}, \delta_{l}, q_{2}\right)(\xi)}(f, \zeta) \neq C_{\Omega\left(\alpha_{k}, \delta_{l}, q_{1}\right)(\xi)}(f, \zeta),
$$

and $\zeta \in E_{\Omega\left(q_{1}\right) \Omega\left(q_{2}\right)}(f)$. Hence $\bigcup_{n, k, l, s, t} A_{n, k, l, s, t} \subset E_{\Omega\left(q_{1}\right) \Omega\left(q_{2}\right)}(f)$.

Equality (5) shows that $E_{\Omega\left(q_{1}\right) \Omega\left(q_{2}\right)}(f)$ is of type $G_{\delta \sigma}$.

It remains to prove that $A=A_{n, k, l, s, t}$ is of porosity $\left(\frac{q_{1}+1}{q_{2}+1}\right)$.

Suppose $A$ is not of porosity $\left(\frac{q_{1}+1}{q_{2}+1}\right)$ at a point $\zeta \in A$. Then for sufficiently small $\varepsilon>0, \Omega\left(\alpha_{s}, \delta_{t}, q_{2}\right)(\zeta) \cap U_{\varepsilon}(\zeta)$ is covered by the set $\bigcup_{\xi \in A} \Omega\left(\alpha_{k}, \delta_{l}\right.$, $\left.q_{1}\right)(\xi)$ by Lemma 1 . Thus if $z \in \Omega\left(\alpha_{s}, \delta_{t}, q_{2}\right)(\zeta) \cap U_{\varepsilon}(\zeta)$, there is a point $\xi \in A$, $z \in \Omega\left(\alpha_{k}, \delta_{l}, q_{1}\right)(\xi)$. Therefore by (4) and (1) $w=f(z)$ lies at a distance $\geqq r_{n}$ from $D_{n}$, and $C_{\Omega\left(a_{s}, \delta_{\ell}, q_{2}\right)(\xi)}(f, \zeta) \cap D_{n}=\phi$. This contradicts $\zeta \in F_{n, s, t}$. Thus the porosity $\left(\frac{q_{1}+1}{q_{2}+1}\right)$ of $A$ is proved.

Remark 1. Dolzhenko's result [3, Theorem 1], then, is for the case $q_{1}=$ 0, $q_{2}=0$, Yanagihara's [4, Theorem 1] for $q_{1}=1, q_{2}=1$, and Yanagihara's [4, Theorem 2] for $q_{1}=0, q_{2}=1$.

Lemma 2. Let $\zeta \in A \subset \Gamma$ and $0 \leqq q$. Suppose $A$ is not of porosity (1) at a point $\zeta \in A$. Then for fixed positive integers $s, t, k, l$ and $m, \Omega\left(\alpha_{s}, \delta_{t}, q\right)(\zeta) \cap U_{\varepsilon}(\zeta)$ is covered by the set $M=\bigcup_{\xi \in A} \nabla\left(\alpha_{k}, \beta_{l}, \delta_{m}, q\right)(\xi)$ supposed $\varepsilon>0$ is sufficiently small.

Proof. Without loss of generality, we may assume that $\zeta=1$. Now we suppose that there exists a sequence $z_{\nu}=r_{\nu} e^{i \theta_{\nu}}(\nu=1,2,3, \cdots)$ such that $z_{\nu} \in \Omega\left(\alpha_{s}, \delta_{t}, q\right)(1), z_{\nu} \notin M$ and $z_{\nu} \rightarrow 1$. For each $z_{\nu}$, points $R_{1}\left(z_{\nu}\right), S_{1}\left(z_{\nu}\right), R_{2}\left(z_{\nu}\right)$ and $S_{2}\left(z_{\nu}\right)$ on $\Gamma$ are decided as follows. 
$R_{1}\left(z_{\nu}\right)$ (or $S_{1}\left(z_{\nu}\right)$ ) is the point on $\Gamma$ such that the point $z_{\nu}$ lies on the curve $t^{+}\left(\alpha_{k}, q\right)\left(R_{1}\left(z_{\nu}\right)\right)$ (or $t^{+}\left(\beta_{l}, q\right)\left(S_{1}\left(z_{\nu}\right)\right)$ ).

$R_{2}\left(z_{\nu}\right)$ (or $S_{2}\left(z_{\nu}\right)$ ) is the point on $\Gamma$ such that the point $z_{\nu}$ lies on the curve $t^{-}\left(\alpha_{k}, q\right)\left(R_{2}\left(z_{\nu}\right)\right)$ (or $t^{-}\left(\beta_{l}, q\right)\left(S_{2}\left(z_{\nu}\right)\right)$ ).

We immediately have

$$
\begin{aligned}
& \left.\overline{R_{1}\left(z_{\nu}\right) S_{1}\left(z_{\nu}\right)} \text { (the arc length } \cdot \text { connecting } R_{1}\left(z_{\nu}\right) \text { and } S_{1}\left(z_{\nu}\right)\right) \\
= & \overline{R_{2}\left(z_{\nu}\right) S_{2}\left(z_{\nu}\right)}=\left(\frac{1-r_{\nu}}{\alpha_{k}}\right)^{\frac{1}{q+1}}-\left(\frac{1-r_{\nu}}{\beta_{l}}\right)^{\frac{1}{q+1}}, \\
& \overline{R_{i}\left(z_{\nu}\right) 1}=\theta_{\nu}+(-1)^{i}\left(\frac{1-r_{\nu}}{\alpha_{k}}\right)^{\frac{1}{q+1}}(i=1,2) .
\end{aligned}
$$

Since $z_{\nu} \in \Omega\left(\alpha_{s}, \delta_{t}, q\right)(1)$, we have $\left|\theta_{\nu}\right|<\left(\frac{1-r_{\nu}}{\alpha_{s}}\right)^{\frac{1}{q+1}}$.

We set

$$
\left.\varepsilon_{\nu}=\max \overline{\left\{R_{1}\left(z_{\nu}\right) 1\right.}, \overline{R_{2}\left(z_{\nu}\right) 1}\right\}
$$

Then we have

$$
\lim _{\nu \rightarrow \infty} \frac{\overline{R_{1}\left(z_{\nu}\right) S_{1}\left(z_{\nu}\right)}}{\left(1-r_{\nu}\right)^{\frac{1}{q+1}}}>0 \text { and } \varepsilon_{\nu}=O\left(\left(1-r_{\nu}\right)^{\frac{1}{q+1}}\right) \text { as } \nu \rightarrow \infty \text {. }
$$

Since $\left[\left\{R_{1}\left(z_{\nu}\right) S_{1}\left(z_{\nu}\right)\right.\right.$ (the arc connecting $R_{1}\left(z_{\nu}\right)$ and $\left.\left.\left.S_{1}\left(z_{\nu}\right)\right)\right\} \cup\left\{R_{2}\left(z_{\nu}\right) S_{2}\left(z_{\nu}\right)\right\}\right] \cap A$ $=\phi$, we have $\gamma\left(1, \varepsilon_{\nu}, A\right) \geqq \overline{R_{1}\left(z_{\nu}\right) S_{1}\left(z_{v}\right)}$. Thus we obtain

$$
\begin{gathered}
\varlimsup_{\varepsilon \rightarrow 0} \frac{1}{\varepsilon} \gamma(1, \varepsilon, A) \geqq \varlimsup_{\nu \rightarrow \infty} \frac{1}{\varepsilon_{\nu}} \overline{R_{1}\left(z_{\nu}\right) S_{1}\left(z_{\nu}\right)} \\
\geqq \varlimsup_{\nu \rightarrow \infty} \frac{\left(1-r_{\nu}\right)^{\frac{1}{q+1}}}{\varepsilon_{\nu}} \frac{\overline{R_{1}\left(z_{\nu}\right) S_{1}\left(z_{\nu}\right)}}{\left(1-r_{\nu}\right)^{\frac{1}{q+1}}}>0,
\end{gathered}
$$

and this contradicts the assumption that 1 is not a point of porosity (1) for $A$. Therefore, for $\varepsilon>0$ small enough, $\Omega\left(\alpha_{s}, \delta_{t}, q\right)(\zeta) \cap U_{\varepsilon}(\zeta)$ is covered by the set $M=\bigcup_{\xi \in A} \nabla\left(\alpha_{k}, \beta_{l}, \delta_{m}, q\right)(\xi)$.

Theorem 2. Let $f: D \rightarrow W$ and $q \geqq 0$. Then $E_{g(q) \nabla(q)}(f)$ is of type $G_{\delta \sigma}$ and of $\sigma$-porosity (1).

Proof. For positive integers $n, k, l$ and $m, E_{n, k, l, m}$ is the set of points $\zeta \in \Gamma$ such that 
the set $\left\{w=f(z) ; z \in \nabla\left(\alpha_{k}, \beta_{l}, \delta_{m}, q\right)(\zeta)\right\}$

lies at a distance $\geqq r_{n}$ from $D_{n}$.

For positive integers $n, s, t$ and $u, F_{n, s, t, u}$ is the set of points such that

the set $\left\{w=f(z) ; z \in \Omega\left(\alpha_{s}, \delta_{t}, q\right)(\zeta), \frac{1}{3 u}<\operatorname{dis}(z, \Gamma)<\frac{1}{u}\right\}$

has common points with $D_{n}$.

Then $E_{n, k, l, m}$ is closed and $F_{n, s, t, u}$ is open. We put

$$
F_{n, s, t}=\bigcap_{i=1}^{\infty} \bigcup_{u=i}^{\infty} F_{n, s, t, u}
$$

and

$$
A_{n, k, l, m, s, t}=E_{n, k, l, m} \cap F_{n, s, t} .
$$

We will show

$$
E_{\Omega(q) \nabla(q)}(f)=\left(\underset{n, k, l, m, s, t}{\cup} A_{n, k, l, m, s, t}\right) \cup E_{\Omega(q) \Omega(q)}(f) .
$$

Take a point $\zeta \in E_{\Omega(q) \nabla(q)}(f)$ and $\zeta \notin E_{\Omega(q) \Omega(q)}(f)$. There exists $\Omega(q)(\zeta)$ and $\nabla(q)(\zeta), \Omega(q)(\zeta) \supset \nabla(q)(\zeta)$, for which $C_{\Omega(q)(\zeta)}(f, \zeta) \supsetneq C_{\nabla(q)(\zeta)}(f, \zeta)$. Choose positive integers $s, t$ and $j$ such that $\Omega\left(\alpha_{s}, \delta_{t}, q\right)(\zeta) \supset \Omega(q)(\zeta)$ and

$$
D_{j} \cap C_{Q\left(\alpha_{s}, \delta_{t}, q\right)(\zeta)}(f, \zeta) \neq \phi, \operatorname{dis}\left(D_{j}, C_{\nabla(q)(\zeta)}(f, \zeta)\right)>5 r_{j} .
$$

Then we can find positive integers $k, l$ and $m$ such that $\nabla(q)(\zeta) \supset \nabla\left(\alpha_{k}, \beta_{l}, \delta_{m}\right.$, $q)(\zeta)$ and $\operatorname{dis}\left(D_{j}, f(z)\right)>4 r_{j}$ for $z \in \nabla\left(\alpha_{k}, \beta_{l}, \delta_{m}, q\right)(\zeta)$. If $D_{n}$ is a disc with radius $r_{n}=2 r_{j}$ and concentric with $D_{j}$, dis $\left(D_{n}, f(z)\right)>r_{n}$ for $z \in \nabla\left(\alpha_{k}, \beta_{l}, \delta_{m}\right.$, $q)(\zeta)$, which shows $\zeta \in E_{n, k, l, m}$. In view of (6) there exists an infinite number of integers $u$ such that

$$
D_{n} \cap\left\{w=f(z) ; z \in \Omega\left(\alpha_{s}, \delta_{t}, q\right)(\zeta), \frac{1}{3 u}<\operatorname{dis}(z, \Gamma)<\frac{1}{u}\right\} \neq \phi,
$$

which shows $\zeta \in F_{n, s, t, u}$. Thus $\zeta \in A_{n, k, l, m, s, t}$ and

$$
E_{\Omega(q) \nabla(q)}(\zeta) \subset\left(\bigcup_{n, k, l, m, s, t} A_{n, k, l, m, s, t}\right) \cup E_{Q(q) \Omega(q)}(f) .
$$

Take a point $\zeta \in\left(\bigcup_{n, k, l, m, s, t} A_{n, k, l, m, s, t}\right) \cup E_{\Omega(q) \Omega(q)}(f)$. If $\zeta \in E_{\Omega(q) \Omega(q)}(f)$, we evidently have $\zeta \in E_{Q(q) \nabla(q)}(f)$. If $\zeta \in \underset{n, k, l, m, s, t}{\cup} A_{n, k, l, m, s, t}$ and $\zeta \in A_{n, k, l, m, s, t}$,

$$
C_{\nabla\left(\alpha_{k}, \beta_{l}, \delta_{m}, q\right)(\xi)}(f, \zeta) \cap D_{n}=\phi \text { from }(1)
$$


and

$$
C_{Q\left(\alpha_{s}, \delta_{t}, q\right)(\xi)}(f, \zeta) \cap D_{n} \neq \phi \text { from }(2)
$$

Thus we have

$$
C_{\nabla\left(\alpha_{k}, \beta_{t}, \delta_{m}, q\right)(\xi)}(f, \zeta) \neq C_{Q\left(\alpha_{s}, \delta_{t}, q\right)(\zeta)}(f, \zeta)
$$

and $\zeta \in E_{\Omega(q) r(q)}(f)$.

Setting $q_{1}=q_{2}=q$ in Theorem 1 , we see that $E_{Q(q) Q(q)}(f)$ is of type $G_{\delta \sigma}$ and of $\sigma$-porosity (1).

Thus the equality (5) shows that $E_{\Omega(q) V(q)}(f)$ is of type $G_{\delta o}$, so that it remains to prove that $A=A_{n, k, l, m, s, t}$ is of porosity (1).

Suppose $A$ is not of porosity (1) at a point $\zeta \in A$. Then for sufficiently small $\varepsilon>0, \Omega\left(\alpha_{s}, \delta_{t}, q\right)(\zeta) \cap U_{\varepsilon}(\zeta)$ is covered by the set $\cup_{\xi \in A} \nabla\left(\alpha_{k}, \beta_{l}, \delta_{m}, q\right)(\xi)$ by Lemma 2. Thus if $z \in \Omega\left(\alpha_{s}, \delta_{t}, q\right)(\zeta) \cap U_{\varepsilon}(\zeta)$, there is a point $\xi \in A, z \in$ $\nabla\left(\alpha_{k}, \beta_{l}, \delta_{m}, q\right)(\xi)$. Therefore by (4) and (1) $w=f(z)$ lies at a distance $\geqq r_{n}$ from $D_{n}$, and $C_{Q\left(\alpha_{s}, \delta_{l}, q\right)(\xi)}(f, \zeta) \cap D_{n}=\phi$. This contradicts $\zeta \in F_{n, s, t}$. Thus that $A$ is of porosity (1) is proved.

3. The following theorem is sharper than Vessey [5, Corollary 2] and Vessey [6, Corollary 1].

Theorem 3. Let $f: D \rightarrow W$ and $0 \leqq q$. Then $E_{\nabla(q) \nabla(q)}(f)$ is of o-porosity (1).

Proof. $E_{\nabla(q) \nabla(q)}(f) \subset E_{\Omega(q) \nabla(q)}(f)$ and $E_{\Omega(q) \nabla(q)}(f)$ is of $\sigma$-porosity (1) by Theorem 2.

Remark 2. Dolzhenko's result [3, Theorem 1], then, is for the case $q=0$, and Yanagihara's [4, Theorem 1] for $q=1$.

Remark 3. Is it true that $\sigma$-porosity (1) of the set $E_{\nabla(q) \nabla(q)}(f)$ is one of its characteristic properties? I would guess that it is positive even for holomorphic functions (see Dolzhenko [3, Theorem 2]).

In the following, the phrase "almost everywhere" means with the exception of a set of linear Lebesque measure 0; and "nearly everywhere" means with the exception of a set of the first Baire category.

Corollary 1. (Vessey [5, Corollary 2;6, Corollary 1]). Let $f: D \rightarrow W$ and $0 \leqq q$. Then almost every and nearly every point of $\Gamma$ belongs to $K_{q}(f)$.

4. Now we can state some generalizations of results in [8]. 
TheOREm 4. Let $f: D \rightarrow W$ and $0 \leqq q_{1}<q_{2}$. Then a $q_{2}$-angular Fatou point of $f(z)$ is a $q_{1}$-angular Fatou point of $f(z)$ except on a set of $\sigma$-porosity (1).

Proof. According to Theorem 2, except on a set of $\sigma$-porosity (1), a $q_{2^{-}}$ angular Fatou point of $f(z)$ is a $q_{2}$-cyclic Fatou point of $f(z)$, which is a $q_{1}$-angular Fatou point.

Theorem 5. Let $f: D \rightarrow W$ and $0 \leqq q_{1}<q_{2}$. Then a $q_{1}$-angular Fatou point of $f(z)$ is a $q_{2}$-angular Fatou point of $f(z)$ except on a set of $\sigma$-porosity $\left(\frac{q_{1}+1}{q_{2}+1}\right)$.

Proof. According to Theorem 2, except on a set of $\sigma$-porosity (1), a $q_{1}$-angular Fatou point of $f(z)$ is a $q_{1}$-cyclic Fatou point. By Theorem 1, except on a set of $\sigma$-porosity $\left(\frac{q_{1}+1}{q_{2}+1}\right)$, a $q_{1}$-cyclic Fatou point of $f(z)$ is a $q_{2}$-cyclic Fatou point of $f(z)$, which is a $q_{2}$-angular Fatou point of $f(z)$.

Theorem 6. Let $f: D \rightarrow W$ and $0 \leqq q_{1}<q_{2}$. Then a $q_{1}$-angular Plessner point of $f(z)$ is a $q_{2}$-angular Plessner point of $f(z)$ except on a set of $\sigma$-porosity (1).

Proof. A $q_{1}$-angular Plessner point of $f(z)$ is a $q_{2}$-cyclic Plessner point of $f(z)$, which is a $q_{2}$-angular Plessner point of $f(z)$ except on a set of $\sigma$-porosity (1) according to Theorem 2.

Theorem 7. Let $f: D \rightarrow W$ and $0 \leqq q_{1}<q_{2}$. Then a $q_{2}$-angular Plessner point of $f(z)$ is a $q_{1}$-angular Plessner point of $f(z)$ except on a set of $\sigma$-porosity $\left(\frac{q_{1}+1}{q_{2}+1}\right)$.

Proof. A $q_{2}$-angular Plessner point of $f(z)$ is a $q_{2}$-cyclic Plessner point of $f(z)$, which is a $q_{1}$-cyclic Plessner point of $f(z)$ except on a set of $\sigma$-porosity $\left(\frac{q_{1}+1}{q_{2}+1}\right)$ according to Theorem 1 .

Remark 4. Is it true that $\sigma$-porosity $\left(\frac{q_{1}+1}{q_{2}+1}\right)$ of the exceptional set of Theorem 5 is one of its characteristic properties? I would guess that it is positive even for holomorphic functions. Whether these conclusions hold for other Theorems 4, 6 and 7 ?

\section{REFERENCES}

[1] Bagemihl, F., Horocyclic boundary properties of meromorphic functions. Ann. Acad. Sci. Fenn., AI, 385, 1-18 (1966).

[2] Dragosh, S., Horocyclic cluster sets of functions defined in the unit disc. Nagoya Math. J. 
35, 53-82 (1969).

[ 3 ] Dolzhenko, E.P., Boundary properties of arbitrary functions. Izv. Akad. Nauk SSSR, 31, 3-14·(1967). English translation: Math. of the USSR IZVESTIJA, 1, 1-12 (1967).

[4] Yanagihara, N., Angular cluster sets and oricyclic cluster sets. Proc. Japan Acad., 45, 423 -428 (1969).

[ 5 ] Vessey, T.A., Tangential boundary behavior of arbitrary functions. Math. Z., 113, 113 $-118(1970)$.

[6] Vessey, T.A., On tangential principal cluster sets of normal meromorphic functions. Nagoya Math. J., 40, 133-137 (1970).

[ 7 ] Collingwood, E.F. and Lohwater, A.J., The Theory of Cluster Sets. Camb. Univ. Press, Cambridge (1966).

[ 8 ] Yoshida, H., Angular Cluster Sets and Horocyclic Angular Cluster Sets. Proc. Japan Acad., 47, 120-125 (1971).

Chiba University, Chiba 